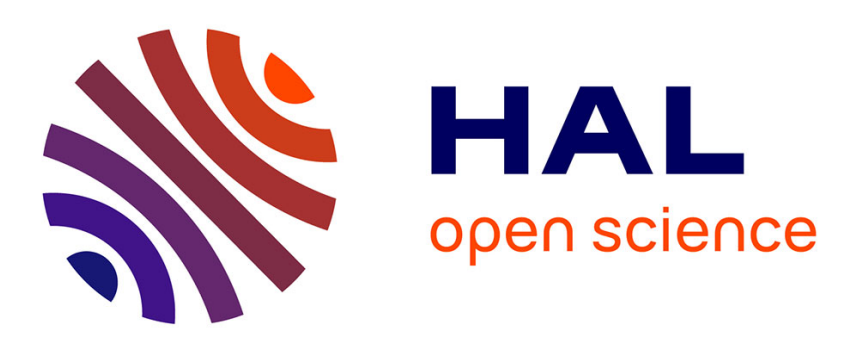

\title{
Analyse d'un schéma de commande pour des couplages haptiques entre le macro-et le nanomonde
} Aude Bolopion, Barthélemy Cagneau, Sinan Haliyo, Stéphane Régnier

\section{To cite this version:}

Aude Bolopion, Barthélemy Cagneau, Sinan Haliyo, Stéphane Régnier. Analyse d'un schéma de commande pour des couplages haptiques entre le macro-et le nanomonde. Journal Européen des Systèmes Automatisés (JESA), 2010, 44 (6), pp.689-716. 10.3166/JESA.44.689-716 . hal-02912310

\section{HAL Id: hal-02912310 https://hal.science/hal-02912310}

Submitted on 5 Aug 2020

HAL is a multi-disciplinary open access archive for the deposit and dissemination of scientific research documents, whether they are published or not. The documents may come from teaching and research institutions in France or abroad, or from public or private research centers.
L'archive ouverte pluridisciplinaire HAL, est destinée au dépôt et à la diffusion de documents scientifiques de niveau recherche, publiés ou non, émanant des établissements d'enseignement et de recherche français ou étrangers, des laboratoires publics ou privés. 


\title{
Analyse d'un schéma de commande pour des couplages haptiques entre le macro- et le nanomonde.
}

\author{
Aude Bolopion* - Barthélemy Cagneau** - D. Sinan Haliyo* - \\ Stéphane Régnier* \\ * Institut des Systèmes Intelligents et de Robotique \\ Université Pierre et Marie Curie, Paris 06 - CNRS UMR 7222 \\ 4 place Jussieu, 75252 Paris Cedex, France \\ \{bolopion,haliyo,regnier\}@isir.fr \\ ** Laboratoire d'Ingénierie des Systèmes de Versailles \\ Université de Versailles Saint-Quentin en Yvelines \\ 45 avenue des Etats-Unis, 78000 Versailles, France \\ barthelemy.cagneau@uvsq.fr
}

\begin{abstract}
RÉSUMÉ. Cet article traite des spécificités de la commande pour des couplages haptiques entre le macro et la nanomonde. La forte homothétie en effort et en déplacement, de même que la particularité du profil des efforts à l'échelle nanoscopique rendent les schémas de commande classiques difficilement utilisables. Une analyse de la stabilité et de la transparence (capacité à restituer de manière fidèle l'impédance de l'environnement) est effectuée en tenant compte de ces spécificités, et l'influence des gains des correcteurs est déterminée. Des opérations d'interaction avec le nanomonde et d'opérations de roulement $2 \mathrm{D}$ avec retour d'effort sont présentées pour valider les conclusions de l'étude théorique.

ABSTRACT. This article deals with the specificity of haptic coupling between macro and nanoworlds. Due to the important homothetic force and displacement factors, as well as the particularities of the force profile at nanoscales, standard control schemes are difficult to use. A stability and transparency (aptitude to reproduce the environment impedance to the user) analysis is carried out considering this specificity. The influence of the controller gains is highlighted. Experiments of felling the nanoworld and $2 D$ rolling operations with force feedback are performed to validate these conclusions.
\end{abstract}

MOTS-CLÉS : téléopération, couplage haptique, commande bilatérale, micromanipulation.

KEYWORDS: teleoperation, haptic coupling, bilateral control, micromanipulation.

JESA - 44/2010. Commande dans le micromonde, pages 689 à 716 


\section{Introduction}

Manipuler des objets de taille nanométrique est actuellement un thème prometteur pour le développement des nanotechnologies (Sitti, 2007). Des domaines aussi variés que la biologie, les sciences des matériaux ou encore l'électronique sont concernés par cette problématique.

Cependant, il subsiste de nombreux problèmes technologiques à cette échelle. En particulier, le manque de vision 3D en temps réel, la précision limitée des outils de manipulation, la faiblesse des rapports signal sur bruit, le manque de répétabilité, la sensibilité des systèmes aux conditions environnementales et la fragilité des objets rendent les opérations de manipulations directes longues et délicates (Sitti, 2001).

La première solution consiste à automatiser les tâches à effectuer. Cette technique demande des modèles fins de l'interaction entre les objets manipulés, les outils utilisés, et l'environnement (Onal et al., 2009). Actuellement, il persiste de nombreuses incertitudes sur les phénomènes nanophysiques. De plus, cette solution interdit toute interactivité entre l'utilisateur et le système au cours de la manipulation. La non-exploitation des connaissances des opérateurs et de leurs capacités de réaction nuit à l'exécution des tâches de manipulation.

L'utilisation d'aides à la manipulation apparaît comme une solution prometteuse, conservant l'interactivité de la manipulation directe tout en augmentant sa facilité. L'haptique est le moyen choisi pour transmettre les informations à l'utilisateur, puisqu'il restitue une indication que les opérateurs ont naturellement l'habitude de traiter lors de manipulations directes à l'échelle macroscopique (Ferreira et al., 2006).

La qualité des informations fournies dépend directement de la stabilité du couplage haptique ainsi que de sa transparence (capacité à restituer de manière fidèle l'impédance de l'environnement). La commande d'un tel système doit donc être considérée en prenant en compte la particularité du profil des efforts dans le nanomonde (dû notamment à la prépondérance des forces surfaciques sur les forces gravitationnelles), ainsi que la différence d'échelle entre le macro- et le nanomonde. Cet article montre dans quelle mesure ces spécificités dégradent la transparence et la stabilité, et comment le choix des gains des correcteurs influe sur le rendu haptique. Des inégalités entre les paramètres sont déterminées pour le réglage de ces différents gains. Des opérations d'interaction avec le nanomonde, et d'opérations de roulement de microbilles avec un retour d'effort bidimensionnel, valident les conclusions.

\section{Spécificités des efforts à l'échelle nanoscopique}

Les difficultés de concevoir un couplage haptique à l'échelle nanoscopique trouvent une partie de leurs origines dans les phénomènes physiques particuliers mis en jeu à cette échelle. Pour appréhender ces derniers, les points caractéristiques d'une courbe de force sont explicités dans cette section. 


\subsection{Système de mesure des efforts}

Une méthode usuelle de mesure des efforts d'interaction à l'échelle nanoscopique utilise la mesure de la déformation, ou déflexion, de l'outil de mesure, appelé aussi poutre ou cantilever. Connaissant la raideur de cet outil par calibrage, l'effort d'interaction est ainsi déduit. Cette poutre peut alors être utilisée comme sonde de mesure et comme outil de manipulation (Sitti, 2004).

Diverses méthodes existent pour mesurer les déformations induites, soit par des jauges intégrées à la base de l'outil, soit par des moyens déportés comme le laser et la réflexion de celui-ci sur la poutre. La mesure de cette réflexion est ainsi effectuée au moyen d'une diode à quadrants, celle-ci mesurant alors la déflexion effective de la poutre (Li et al., 2004). Seul le principe de mesure par laser est détaillé dans cette soussection, puisqu'il s'agit de celui disponible sur la plate-forme utilisée pour réaliser les expériences.

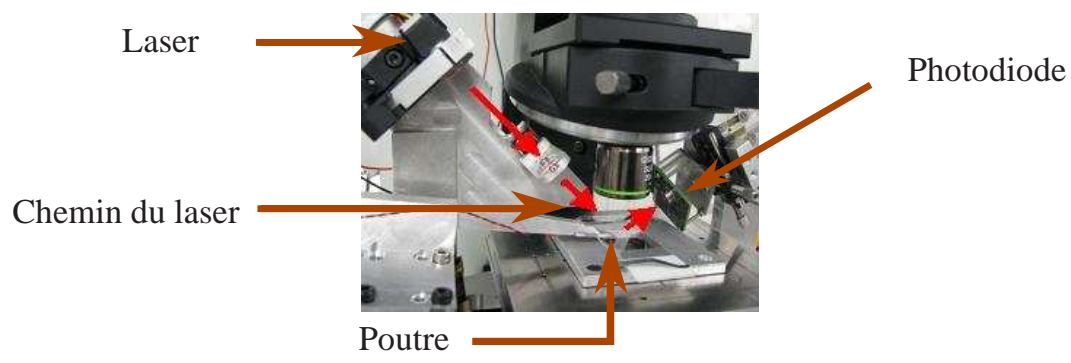

Figure 1. Système de mesure des efforts par laser

La figure 1 représente le système de mesure, où le rayon laser est réfléchi par la poutre sur la photodiode. Cette dernière est composée de quatre quadrants de tensions respectives $T_{l}, T_{r}, B_{l}, B_{r}$. Deux tensions de sortie sont disponibles, $u_{v}$ et $u_{h}$, définies comme :

$$
\begin{aligned}
& u_{v}=\left(T_{l}+T_{r}\right)-\left(B_{l}+B_{r}\right) \\
& u_{h}=\left(T_{l}+B_{l}\right)-\left(T_{r}+B_{r}\right)
\end{aligned}
$$

Elles correspondent à une mesure du déplacement de l'impact du laser sur la photodiode selon les axes respectivement vertical et horizontal. La position de cet impact variant en fonction des déformations de la poutre, il est possible, à partir des signaux de sortie de la photodiode, de mesurer la déflexion et la torsion de la poutre (cf. soussection 4.2.1). Connaissant le point d'application de la force, son module est déduit.

Pour étudier les caractéristiques du profil des efforts, un cas simple est traité : une force verticale est appliquée sur la ligne médiane de la poutre en son extrémité. Le laser est supposé être focalisé sur ce point. En utilisant la mesure de tension correspondant à l'axe vertical $u_{v}$, et la sensibilité de la photodiode $S_{v}$, la force $F_{e}$ appliquée par le substrat sur la poutre est :

$$
F_{e}=k_{z} \delta=k_{z} S_{v} u_{v}
$$


où les variables sont :

$-\delta$ : déflexion de la poutre

$-S_{v}:$ sensibilité de la photodiode - calibrée

$-u_{v}$ : tension de sortie de la photodiode - mesurée

$-k_{z}$ : raideur de la poutre (de quelques centièmes à plusieurs dizaines de $\mathrm{N} \cdot \mathrm{m}^{-1}$ ) - calibrée. Il convient de noter que la raideur a également pour unités le $\mu \mathrm{N} \cdot \mathrm{m}^{-1}$ ou le $\mathrm{nN} \cdot \mathrm{m}^{-1}$, qui sont plus adaptées pour décrire les phénomènes aux échelles nanoscopiques.

Le calibrage de la poutre est réalisé en utilisant l'expression de la raideur en fonction des dimensions de la poutre :

$$
k_{z}=\frac{E w t^{3}}{4 L^{3}}
$$

où $E$ est le module d'Young, $w, L$ et $t$ sont respectivement les largeur, longueur et épaisseur de la poutre. Les deux premières dimensions sont estimées à l'aide d'un microscope optique. Pour mesurer précisément l'épaisseur la poutre est excitée, et les fréquences de résonance sont enregistrées pour les différents modes propres. L'épaisseur de la poutre est alors calculée d'après :

$$
t=\frac{w_{n}}{K n^{2}} \sqrt{\frac{12 \rho}{E}}
$$

où $w_{n}$ est la fréquence de résonance en flexion du $n^{i e m e}$ mode propre, $K_{n}$ est le nombre d'onde de la poutre et $\rho$ est sa masse volumique. Cette méthode est décrite dans (Xie et al., 2008).

L'équation [2] montre que la force mesurée dépend de la raideur de la poutre. Ce paramètre est susceptible de varier significativement selon la tâche effectuée. En effet, des poutres raides sont utilisées pour interagir de manière précise avec les objets lors d'opérations de manipulation. Les poutres plus souples peuvent mesurer des forces plus faibles, et sont donc adaptées pour l'exploration. Le couplage haptique doit prendre en compte cette particularité.

\subsection{Courbes d'approche-retrait}

Afin de déterminer les caractéristiques des efforts dans le nanomonde, une manipulation 1D consistant à approcher la poutre du substrat, appliquer une force, et à la retirer du substrat est réalisée. La force appliquée sur la poutre au cours d'une telle expérience est représentée sur la figure $2 \mathrm{a}$ en fonction de la distance entre la poutre et le substrat (les flèches indiquent le déroulement chronologique). L'évolution des efforts en fonction du temps correspondant à la même manipulation est donnée figure 2b. Cette courbe représente ce que l'opérateur doit ressentir au cours de l'expérience via l'interface haptique. 


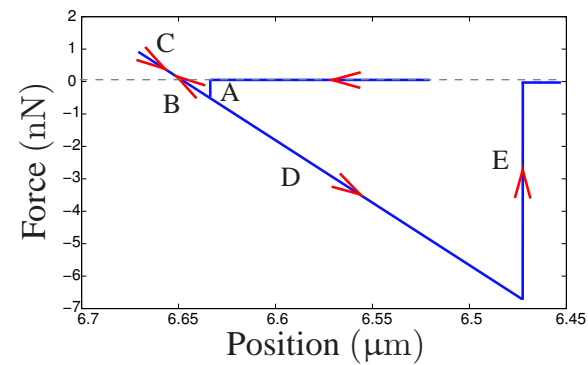

(a) force en fonction de la position

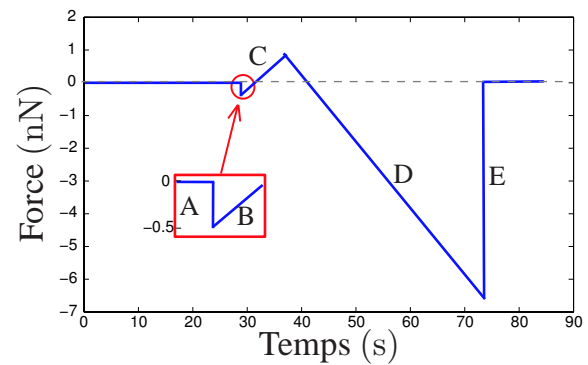

(b) force en fonction du temps

Figure 2. Force appliquée sur la poutre lors d'une expérience d'approche-retrait

Plusieurs caractéristiques apparaissent sur ces courbes d'effort :

- La présence de deux phénomènes discontinus (notés A et $\mathrm{E}$ sur les tracés). Ils représentent respectivement les instants pendant lesquels la poutre est attirée (pull-in) puis relâchée (pull-off) par le substrat. Dans ces deux cas, ces phénomènes indiquent des changements d'équilibre de l'interaction poutre-substrat. Par exemple, pour la rupture, la force élastique de la poutre est supérieure à la force de contact.

- La variété des forces d'interaction. Les premiers efforts appliqués sur la poutre au cours de la manipulation (B) ainsi que les derniers (D) sont attractifs, alors que ceux ayant lieu entre ces instants sont répulsifs (C).

- La variation de l'amplitude des efforts. L'amplitude des forces de pull-in est 10 à 100 fois moins importante que celle du pull-off.

- La faible amplitude de la force de pull-in. Elle est en effet de l'ordre de quelques dizaines de nanonewtons.

Le schéma de couplage doit transmettre à l'utilisateur de manière la plus fidèle possible l'ensemble de ces phénomènes.

\section{Analyse du schéma de commande force-position dans le contexte de la nanorobotique}

Dans cette section, un schéma de commande classique, le couplage force-position $(F P)$ est étudié dans le contexte de la nanorobotique (voir figure 3). Dans toute la suite, $H(s)$ (respectivement $H(z)$ ) fait référence à la fonction de transfert continue (respec- 
tivement discrète) correspondant à l'interface haptique. Cette dernière est modélisée par un corps rigide, d'inertie $M_{h}$ et d'amortissement $B_{h}$ :

$$
\begin{aligned}
& H(s)=\frac{V_{m}(s)}{F_{o p}(s)+F_{m}(s)}=\left[\left(B_{h}+M_{h} s\right)\right]^{-1} \\
& H(z)=\frac{V_{m}(z)}{F_{o p}(z)+F_{m}(z)}=\left(1-z^{-1}\right) Z\left\{\frac{H(s)}{s}\right\}=\frac{1}{B_{h}} \frac{1-\delta}{z-\delta}
\end{aligned}
$$

où $s$ représente la variable de Laplace, $z$ est la variable temporelle discrète, $Z\{$.$\} est$ l'opérateur de transformée en $\mathrm{Z}$ et $\delta=e^{-\frac{B_{h} T_{e}}{M_{h}}}$.

Le robot esclave qui manipule la poutre par rapport au substrat (nanotranslateur) est représenté par une fonction de transfert comprenant deux constantes de temps $\tau_{1}$ et $\tau_{2} . N(s)$ (respectivement $N(z)$ ) est sa fonction de transfert continue (respectivement discrète) en boucle fermée :

$$
\begin{aligned}
& N(s)=\frac{V_{c}(s)}{V_{s}(s)}=\left[\left(1+\tau_{1} s\right)\left(1+\tau_{2} s\right)\right]^{-1} \\
& N(z)=\frac{V_{c}(z)}{V_{s}(z)}=1-\alpha_{1} \frac{z-1}{z-e^{-\frac{T_{e}}{\tau_{1}}}}-\alpha_{2} \frac{z-1}{z-e^{-\frac{T_{e}}{\tau_{2}}}}
\end{aligned}
$$

où $\alpha_{1}=\frac{1}{1-\frac{\tau_{2}}{\tau_{1}}}$ et $\alpha_{2}=\frac{1}{1-\frac{\tau_{1}^{1}}{\tau_{2}}}$.

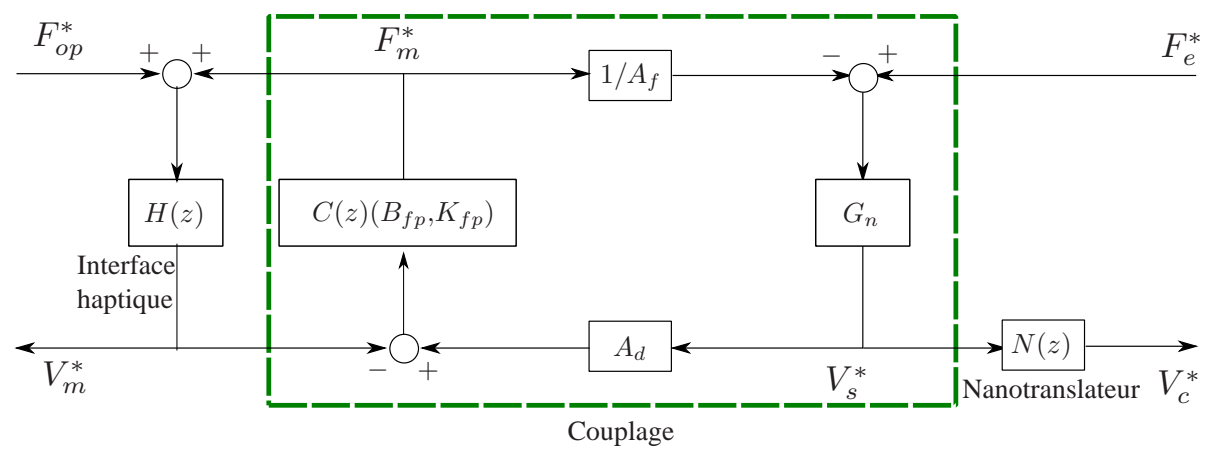

Figure 3. Schéma de commande force-position

Les entrées de ce schéma de couplage sont la vitesse de l'interface haptique $V_{m}$ et la force appliquée par l'environnement sur la poutre $F_{e}$ (équation [2]). Les sorties sont la vitesse utilisée comme entrée du robot esclave $V_{s}$ et la force retournée à l'utilisateur par l'interface haptique $F_{m}$. A cause des différences d'échelle entre le macroet le micromonde, il est nécessaire de lier les grandeurs (forces et déplacements) de l'interface haptique et celles des objets manipulés. Le coefficient homothétique en effort (respectivement déplacement) est noté $A_{f}$ (respectivement $A_{d}$ ). L'exposant étoile 
$\left({ }^{*}\right)$ indique les paramètres discrets. Les deux correcteurs $G_{n}$ et $C$ sont respectivement des correcteurs proportionnel $(P)$ et proportionnel-intégral $(P I)$. $C$ est composé d'une raideur $K_{f p}$ et d'un amortissement $B_{f p}$. Il est discrétisé en utilisant l'approximation de Tustin :

$$
\begin{aligned}
& C(s)=B_{f p}+\frac{K_{f p}}{s} \\
& C(z)=B_{f p}+K_{f p} \frac{T_{e}(z+1)}{2(z-1)}
\end{aligned}
$$

Le retour de force $F_{m}$ est calculé à l'aide du correcteur PI. Le terme intégral $K_{f p}$ et le gain proportionnel $B_{f p}$ sont utilisés pour modifier la raideur et l'amortissement de la force retournée. Le gain $G_{n}$ est utilisé pour calculer la vitesse de consigne du nanotranslateur (robot esclave qui manipule la poutre par rapport au substrat).

Dans les sections suivantes, ce schéma de couplage est étudié en termes de transparence et de stabilité. Lorsqu'une modélisation de la relation liant la position de consigne de la poutre aux forces d'interaction est nécessaire, l'hypothèse 1 est faite :

Hypothèse 1. Le robot esclave interagit avec l'environnement à l'aide d'une poutre de raideur $k_{z}$. Le substrat est modélisé par une raideur $k_{s}$. Ces deux ressorts en série forment un ressort équivalent de raideur $k_{e}$ :

$$
\frac{1}{k_{e}}=\frac{1}{k_{s}}+\frac{1}{k_{z}}
$$

Le cas le plus défavorable pour la stabilité est de considérer la raideur la plus élevée pour le ressort équivalent $k_{e}$. Prendre $k_{e}=k_{z}$ revient donc à se placer dans un cas restrictif. Cette approximation, bien que forte, est utile pour mettre en évidence l'influence de l'environnement sur les performances du système, et est utilisée dans la suite de l'article.

\subsection{Etude de la transparence}

La transparence d'un couplage haptique est sa capacité à retransmettre fidèlement à l'opérateur les phénomènes du nanomonde. Cette propriété est définie comme une comparaison entre l'impédance ressentie par l'utilisateur $Z_{o p}=\frac{F_{o p}}{V_{m}}$ et celle de l'environnement $Z_{e}=\frac{-F_{e}}{V_{c}}$ (Hokayem et al., 2006) et (Lawrence, 1993). La transparence idéale est atteinte quand :

$$
Z_{o p}=Z_{e}
$$

Cependant, aux échelles micro- et nanoscopique il convient de prendre en compte les facteurs d'échelle en force $A_{f}$ et en déplacement $A_{d}$ :

$$
\frac{F_{o p}}{V_{m}}=\frac{-A_{f} F_{e}}{A_{d} V_{c}} \Leftrightarrow Z_{o p}=\frac{A_{f}}{A_{d}} Z_{e}
$$


Il faut bien noter que cette définition suppose que les efforts sont mesurés fidèlement. Or, comme cela a été vu dans la sous-section 2.1, les forces mesurées sont filtrées par la sonde utilisée.

Pour analyser la transparence, l'impédance ressentie par l'utilisateur $Z_{o p}=$ $F_{o p} / V_{m}$ est calculée en utilisant le schéma de commande représenté figure 3 , et les fonctions de transfert continues :

$$
Z_{o p}=\frac{n_{z_{e}} Z_{e}+A_{f} C(s) H(s)+A_{f}+A_{d} G_{n} C(s)}{d_{z_{e}} Z_{e}+A_{f} H(s)+A_{d} G_{n} C(s) H(s)}
$$

où :

$$
\begin{aligned}
n_{z_{e}} & =A_{f} G_{n} C(s) H(s) N(s)+A_{f} G_{n} N(s) \\
d_{z_{e}} & =A_{f} G_{n} H(s) N(s)
\end{aligned}
$$

Deux cas particuliers, le mode contact (la poutre et le substrat sont en contact) et le mode non-contact (aucun effort n'est appliqué sur la poutre) sont étudiés dans les sous-sections suivantes. Les comportements en basses et hautes fréquences sont mis en évidence.

\subsubsection{Contact}

Quand le contact est effectif, $Z_{o p}^{B F}$ en basses fréquences est :

$$
Z_{o p}^{B F} \underset{\omega<<1}{\approx} \frac{A_{f}}{A_{d}+\frac{A_{f} k_{e}}{K_{f p}}} Z_{e}+\frac{1}{1+\frac{A_{d} K_{f p}}{A_{f} k_{e}}}+\frac{1}{\frac{G_{n} k_{e}}{K_{f p}}+\frac{A_{d} G_{n}}{A_{f}}}+B_{h}
$$

L'impédance de l'environnement est divisée par $\frac{A_{f}}{A_{d}+\frac{A_{f} k_{e}}{K_{f p}}}$. Si $K_{f p}$ augmente, ce terme tend vers l'impédance idéale. Le terme $\frac{1}{1+\frac{A_{d} K_{f p}}{A_{f} k_{e}}}+\frac{\frac{1}{G_{n} k_{e}}+\frac{A_{d} G_{n}}{A_{f}}}{K_{f p}}$ et la viscosité de l'interface haptique ajoutent une erreur sur l'impédance ressentie par l'utilisateur. Cette erreur peut être minimisée si $K_{f p}$ et $G_{n}$ sont suffisamment grands.

Pour les hautes fréquences, l'impédance $Z_{o p}^{H F}$ ressentie par l'utilisateur est :

$$
Z_{o p}^{H F} \underset{\omega>>1}{\approx} M_{h} j \omega
$$

L'utilisateur ressent principalement l'inertie de l'interface haptique pour $\omega>>1$.

Le diagramme de Bode représenté sur la figure 4a est utile pour illustrer les résultats analytiques et valider les approximations.

Remarque. - Les diagrammes de Bode (figure 4) ont été tracés en utilisant les valeurs numériques des expériences sous-section $4.1: k_{e}=2,4 \mathrm{~N} \cdot \mathrm{m}^{-1}$, $A_{f}=6 \cdot 10^{6}, A_{d}=0,05 \cdot 10^{6}, K_{f p}=100 \mathrm{~N} \cdot \mathrm{m}^{-1}, B_{f p}=2 \mathrm{~N} \cdot \mathrm{s} \cdot \mathrm{m}^{-1}$ et 
$G_{n}=48,0 \mathrm{~m} \cdot \mathrm{N}^{-1} \cdot \mathrm{s}^{-1}$, ainsi que les paramètres de l'interface haptique et du nanotranslateur présentés section 4. Il faut noter que ces valeurs sont définies uniquement pour illustrer les résultats, mais ne modifient en rien les conclusions tirées.

En basses fréquences, les variations du tracé de la phase du diagramme de Bode sont identiques pour l'impédance du contact simulé et pour celle de l'environnement. Les impédances réelles et ressenties diffèrent seulement d'une constante dont la valeur peut être réduite en augmentant $K_{f p}$ et $G_{n}$. Ainsi, quand l'utilisateur atteint le point de contact, il est capable de détecter la variation des efforts présents au cours de la manipulation.

\subsubsection{Non-contact}

Lorsqu'aucune force ne s'applique sur la poutre, l'impédance ressentie par l'utilisateur est :

$$
Z_{o p}=\frac{A_{f}\left(K_{f p}+B_{f p} j \omega\right)}{A_{f} j \omega+A_{d} G_{n}\left(K_{f p}+B_{f p} j \omega\right)}+M_{h} j \omega+B_{h}
$$

En basses fréquences, l'impédance $Z_{o p}^{B F}$ peut être approchée par :

$$
Z_{o p}^{B F} \underset{\omega<<1}{\approx} \frac{A_{f}}{A_{d} G_{n}}+B_{h}
$$

Pour réduire l'impédance ressentie par l'utilisateur sans modifier les facteurs d'échelle, $G_{n}$ est le seul paramètre pouvant être réglé. Plus il sera élevé, meilleure sera la transparence. Cependant, quelles que soient les valeurs des correcteurs, l'utilisateur ressentira la viscosité de l'interface haptique.

En hautes fréquences, il ressentira l'impédance $Z_{o p}^{H F}$ :

$$
Z_{o p}^{H F} \underset{\omega>>1}{\approx} M_{h} j \omega
$$

Comme pour le mode contact, l'opérateur ressentira l'inertie de l'interface haptique.

Le diagramme de Bode (figure 4b) confirme la validité des approximations faites sur les équations [20] et [21]. Le manque de transparence en basses fréquences peut être réduit en augmentant $G_{n}$.

Le tableau 1 résume les impédances transmises à l'utilisateur. Pour obtenir une transparence approchant la transparence idéale, $K_{f p}$ doit être suffisamment élevé pour assurer un contact raide, alors que $G_{n}$ influe sur le comportement en modes contact et non-contact. Plus ce gain est élevé, et moins le ressenti est visqueux.

\subsection{Etude de la stabilité}

L'analyse de stabilité est effectuée à partir des fonctions de transfert discrètes car les intégrations numériques de ce schéma le rendent sensible à la période d'échan-

tillonnage $T_{e}$. Compte tenu de la complexité du schéma de commande, il n'est pas 
possible d'appliquer un critère de stabilité comme celui de Routh-Hurwitz pour obtenir des relations simples mettant en évidence l'influence de chacun des gains du couplage pour un environnement donné. La stabilité du couplage peut être étudiée

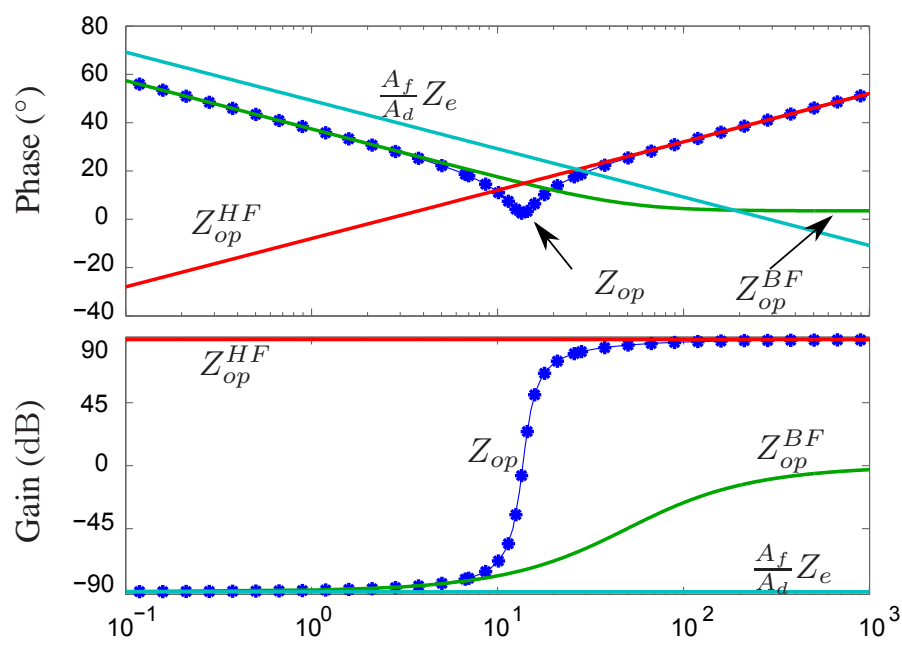

Pulsation $\omega(\mathrm{rad} / \mathrm{s})$

(a) mode contact
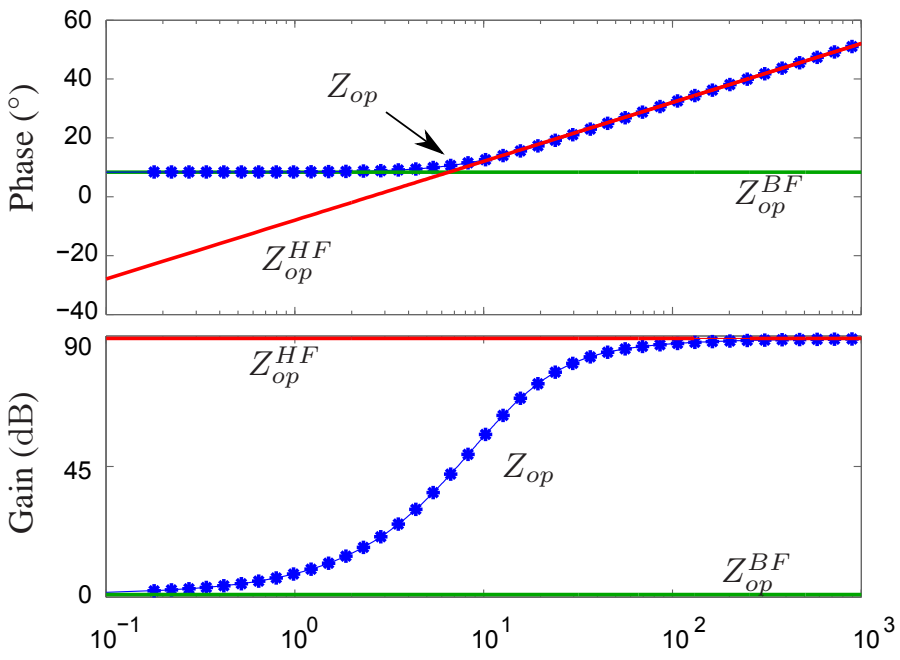

Pulsation $\omega(\mathrm{rad} / \mathrm{s})$

(b) mode non-contact

Figure 4. Diagrammes de Bode des impédances du schéma de couplage FP 


\begin{tabular}{|l|c|c|}
\cline { 2 - 3 } \multicolumn{1}{c|}{} & $\begin{array}{c}\text { Non- } \\
\text { contact }\end{array}$ & Contact \\
\hline Basses fréquences & $\frac{A_{f}}{A_{d} G_{n}}+B h$ & $\frac{A_{f}}{A_{d}+\frac{A_{f} k_{e}}{K_{f p}}} Z_{e}+\frac{1}{1+\frac{A_{d} K_{f p}}{A_{f} k_{e}}}+\frac{1}{\frac{G_{n} k_{e}}{K_{f p}}+\frac{A_{d} G_{n}}{A_{f}}}+B_{h}$ \\
\hline Hautes fréquences & \multicolumn{2}{|c|}{$M_{h} j \omega$} \\
\hline
\end{tabular}

Tableau 1. Valeurs approchées de $Z_{o p}$

sans modéliser l'environnement en se basant sur la notion de passivité (Khalil, 2002). Cette approche est néanmoins très conservative puisque seule l'hypothèse d'un opérateur et d'un environnement passifs est faite. L'environnement et l'opérateur étant des systèmes à un port uniquement (la passivité considère le cas général de systèmes quelconques à deux ports), un critère moins conservatif, la stabilité inconditionnelle (Adams et al., 1999), est utilisée. Les conditions de stabilité mises en évidence dans cette sous-section à l'aide du critère de Llewelyn seront testées avec les valeurs numériques choisies selon les relations déterminées dans la sous-section 3.3.

La matrice admittance du couplage $\mathbf{P}$ est définie par :

$$
\begin{aligned}
{\left[\begin{array}{c}
V_{m}^{*} \\
V_{c}^{*}
\end{array}\right] } & =\mathbf{P}(z) \cdot\left[\begin{array}{c}
F_{o p}^{*} \\
F_{e}^{*}
\end{array}\right] \\
& =\left[\begin{array}{ll}
p_{11}(z) & p_{12}(z) \\
p_{21}(z) & p_{22}(z)
\end{array}\right] \cdot\left[\begin{array}{c}
F_{o p}^{*} \\
F_{e}^{*}
\end{array}\right]
\end{aligned}
$$

où :

$$
\begin{aligned}
& p_{11}(z)=H(z)\left[A_{f}+A_{d} G_{n} C(z)\right] / D(z) \\
& p_{12}(z)=\left[A_{f} A_{d} G_{n} C(z) H(z)\right] / D(z) \\
& p_{21}(z)=\left[G_{n} H(z) C(z)\right] / D(z) \\
& p_{22}(z)=A_{f} G_{n}[1+C(z) H(z)] / D(z) \\
& D(z)=A_{f}+A_{d} G_{n} C(z)+A_{f} H(z) C(z)
\end{aligned}
$$

Le critère de Llewelyn est vérifié si et seulement si les conditions $C_{1}, C_{2}$ et $C_{3}$ suivantes sont respectées (Llewellyn, 1952) :

$$
\begin{aligned}
C_{1}=\operatorname{Re}\left(p_{11}\right) & \geq 0 \\
C_{2}=\operatorname{Re}\left(p_{22}\right) & \geq 0 \\
C_{3}=2 \operatorname{Re}\left(p_{11}\right) \operatorname{Re}\left(p_{22}\right)-\left|p_{12} p_{21}\right|-\operatorname{Re}\left(p_{12} p_{21}\right) & \geq 0
\end{aligned}
$$

Ces inégalités seront utilisées pour vérifier que les gains choisis sont conformes aux conditions de stabilité. 


\subsection{Détermination des gains des correcteurs}

La qualité de ce schéma de couplage dépend des paramètres des correcteurs utilisés, et des facteurs d'échelle (cf. sous-sections 3.1 et 3.2). Cependant, les relations mises en évidence dans les sections précédentes ne permettent pas de choisir facilement les gains puisque chacune d'entre elles est composée de plusieurs paramètres. Dans les sous-sections suivantes, des cas particuliers du schéma sont donc considérés pour obtenir des relations simples sur des conditions nécessaires de stabilité. En utilisant ces relations, ainsi que les considérations sur la transparence, les gains sont choisis. La stabilité du système est alors vérifiée en utilisant le critère de Llewelyn.

\subsubsection{Facteurs d'échelle}

$A_{f}$ et $A_{d}$ sont choisis d'après les besoins de l'utilisateur. Selon les tâches, une manipulation précise et/ou une forte amplification des efforts sont nécessaires. $A_{d}$ est sélectionné selon l'espace de travail de l'interface haptique, et la précision du déplacement souhaitée. $A_{f}$ dépend de la poutre utilisée. Sa valeur est choisie de telle sorte que l'amplitude des forces retournées à l'utilisateur soit suffisante pour qu'un opérateur non entraîné puisse distinguer facilement les différents phénomènes ayant lieu pendant l'expérience.

\subsubsection{Correcteur proportionnel $G_{n}$}

Les problèmes d'instabilité peuvent être dus à la structure des schémas de couplage, mais aussi aux calculs numériques. En effet, la force $F_{m}^{*}$ à l'instant $k+1$ est déterminée en utilisant les informations de position et de vitesse à l'instant $k$ (cf. figure 3) :

$$
F_{m}^{*}(k+1)=B_{f p} \Delta V(k)+K_{f p} \Delta X(k)
$$

où :

$$
\begin{aligned}
& \Delta V(k)=A_{d} V_{s}^{*}(k)-V_{m}^{*}(k) \\
& \Delta X(k)=A_{d} X_{s}^{*}(k)-X_{m}^{*}(k)
\end{aligned}
$$

Pour des raisons similaires, l'expression de $V_{s}^{*}$ est :

$$
V_{s}^{*}(k+1)=G_{n}\left[F_{e}^{*}(k)-\frac{1}{A_{f}} F_{m}^{*}(k)\right]
$$

En considérant [26] et [28], et le fait que la position $X_{s}^{*}$ soit calculée en utilisant l'approximation de Tustin lorsque la poutre est loin du substrat (aucune force appliquée sur la poutre i.e. $\left.F_{e}^{*}=0\right), F_{m}^{*}$ est donnée par:

$$
F_{m}^{*}(k+1)=\lambda_{1} F_{m}^{*}(k-1)+\lambda_{2} V_{m}^{*}(k)+\left(\lambda_{3}+\lambda_{4}\right) K_{f p}
$$


où :

$$
\begin{aligned}
& \lambda_{1}=-G_{n}\left[\frac{B_{f p} A_{d}+K_{f p} A_{d} \frac{T_{e}}{2}}{A_{f}}\right] \\
& \lambda_{2}=-\left[B_{f p}+K_{f p} \frac{T_{e}}{2}\right] \\
& \lambda_{3}=A_{d}\left[X_{s}^{*}(k-1)+\frac{V_{s}^{*}(k-1) T_{e}}{2}\right] \\
& \lambda_{4}=-\left[X_{m}^{*}(k-1)+\frac{V_{m}^{*}(k-1) T_{e}}{2}\right]
\end{aligned}
$$

Pour que les calculs numériques ne divergent pas, $G_{n}$ doit être inférieur à une valeur maximale (condition de stabilité nécessaire) :

$$
\left|\lambda_{1}\right|<1 \Leftrightarrow G_{n}<\frac{A_{f}}{A_{d} K_{f p} \frac{T_{e}}{2}+A_{d} B_{f p}}=G_{n_{l i m}}
$$

\subsubsection{Correcteur proportionnel-intégral : $B_{f p}$ et $K_{f p}$}

Les travaux de (Gil et al., 2004) présentent une relation entre $B_{f p}, K_{f p}$ et $T_{e} . \mathrm{Si}$ cette dernière est vérifiée, alors la stabilité du système est assurée lors d'une phase de contact avec un environnement rigide. Dans cette étude, les auteurs appliquent le critère de Routh-Hurwitz à un schéma de commande similaire à celui considéré dans ce document. Cependant, la sortie du couplage est une position $\left(X_{m}\right)$, au lieu d'une vitesse $\left(V_{m}\right)$ dans notre exemple. De plus, la méthode Euler implicite est utilisée pour déterminer le correcteur discret $C(z)$ (au lieu de Tustin).

La même démarche est appliquée ici. Le système considéré est linéaire, invariant dans le temps. Sa fonction de transfert discrète est :

$$
\frac{V_{m}^{*}}{F_{o p}^{*}}=\frac{H(z)}{1+H(z) C(z)}
$$

Avant de pouvoir appliquer le critère de Routh-Hurwitz sur l'équation caractéristique, une transformation bilinéaire $\left(z=\frac{2+w T_{e}}{2-w T_{e}}\right)$ doit être effectuée. L'équation obtenue est alors :

$$
b_{2} w^{2}+b_{1} w+b_{0}=0
$$

où :

$$
\begin{aligned}
& b_{2}=4 B_{h}(1+\delta)-4(1-\delta) B_{f p} \\
& b_{1}=(1-\delta)\left(4 B_{h}+4 B_{f p}-2 K_{f p} T_{e}\right) \\
& b_{0}=2 K_{f p} T_{e}(1-\delta)
\end{aligned}
$$


Le critère de Routh-Hurwitz est vérifié si et seulement si $b_{0}, b_{1}$ et $b_{2}$ sont du même signe. Comme $\delta<1$, l'équation [35] est toujours positive. Le système sera donc stable si et seulement si $b_{1}>0$ et $b_{2}>0$, ce qui implique :

$$
\begin{aligned}
B_{h}+B_{f p} & >\frac{K_{f p} T_{e}}{2} \\
B_{h} \frac{1+\delta}{1-\delta} & >B_{f p}
\end{aligned}
$$

L'équation [36] est la même condition que celle trouvée dans (Gil et al., 2004) et signifie que la raideur du couplage est limitée par la viscosité de l'interface haptique et celle ajoutée par le couplage. De plus, pour une viscosité donnée, si la période d'échantillonnage augmente, $K_{f p}$ doit diminuer pour vérifier les conditions de stabilité.

Le paramètre $\delta$ de l'équation [37] est une caractéristique de l'interface haptique (voir équation [6] $: \delta=e^{-\frac{B_{h} T_{e}}{M_{h}}}$ ). En utilisant un développement de Taylor à l'ordre 1 de $x \mapsto e^{x}$ autour de 0 ( $T_{e}$ tend vers 0 ), $\delta \approx 1-\frac{B_{h} T_{e}}{M_{h}}$. L'équation [37] peut être approchée par :

$$
\frac{2 M_{h}}{T_{e}}>B_{h}+B_{f p}
$$

Cette équation met en évidence que la viscosité maximale (et par conséquent, d'après l'équation [36], la raideur maximale) admissible est limitée par l'inertie du bras maître et est inversement proportionnelle à la période d'échantillonnage $T_{e}$. Cette propriété met en évidence l'importance de la période d'échantillonnage pour garantir un retour d'effort stable.

\subsubsection{Résumé}

Les relations mises en évidence section 3.1 ainsi que celles des sous-sections 3.3.2 et 3.3.3 sont résumées dans le tableau 2. Les valeurs minimales et maximales que chaque gain peut prendre tout en vérifiant les conditions de stabilité et de transparence sont indiquées.

Comme montré aux sous-sections 3.1.1 et 3.1.2, $G_{n}$ doit être élevé pour obtenir un système transparent en phase de contact et de non-contact. D'après [30], pour des raisons de stabilité, $G_{n}$ doit être inférieur à $\frac{A_{f}}{A_{d} K_{f p} \frac{T_{e}}{2}+A_{d} B_{f p}}$. Donc diminuer $B_{f p}$ et $K_{f p}$ donne une valeur $G_{n_{l i m}}$ plus élevée, et augmente la transparence.

$K_{f p}$ est limité par une valeur maximale pour vérifier le critère de Routh-Hurwitz. Cependant, comme expliqué précédemment, pour obtenir la meilleure transparence possible, $K_{f p}$ doit être suffisamment faible pour limiter la viscosité dans les phases de non-contact par l'intermédiaire du paramètre $G_{n_{l i m}}$. 


\begin{tabular}{|c|c|c|}
\cline { 2 - 3 } \multicolumn{1}{c|}{} & Valeur minimale & Valeur maximale \\
\hline$K_{f p}$ & $\begin{array}{c}\text { haute pour garantir un ressenti du } \\
\text { contact raide (cf. 3.1.1) }\end{array}$ & {$[36],[38] \rightarrow \frac{4 M_{h}}{T_{e}^{2}}$} \\
\hline$B_{f p}$ & {$[36] \rightarrow \frac{K_{f p} T_{e}}{2}-B_{h}$} & $\begin{array}{c}\text { faible pour garantir la } \\
\text { transparence en non-contact } \\
\text { (cf. 3.1.2) }\end{array}$ \\
\hline$G_{n}$ & $\begin{array}{c}\text { élevée pour garantir la transparence } \\
\text { en contact et non-contact (cf. 3.1.1 } \\
\text { et 3.1.2) }\end{array}$ & {$[30] \rightarrow \frac{A_{f}}{A_{d} K_{f p} \frac{T_{e}}{2}+A_{d} B_{f p}}$} \\
\hline
\end{tabular}

Tableau 2. Valeurs admissibles pour les gains des correcteurs

A l'aide de ces relations sur les gains, le réglage du système est réalisé pour deux expériences présentées dans la section suivante : une opération d'interaction avec le nanomonde et une opération de roulement avec un retour d'effort bidimensionnel.

\section{Exemple de téléopérations avec retour d'effort}

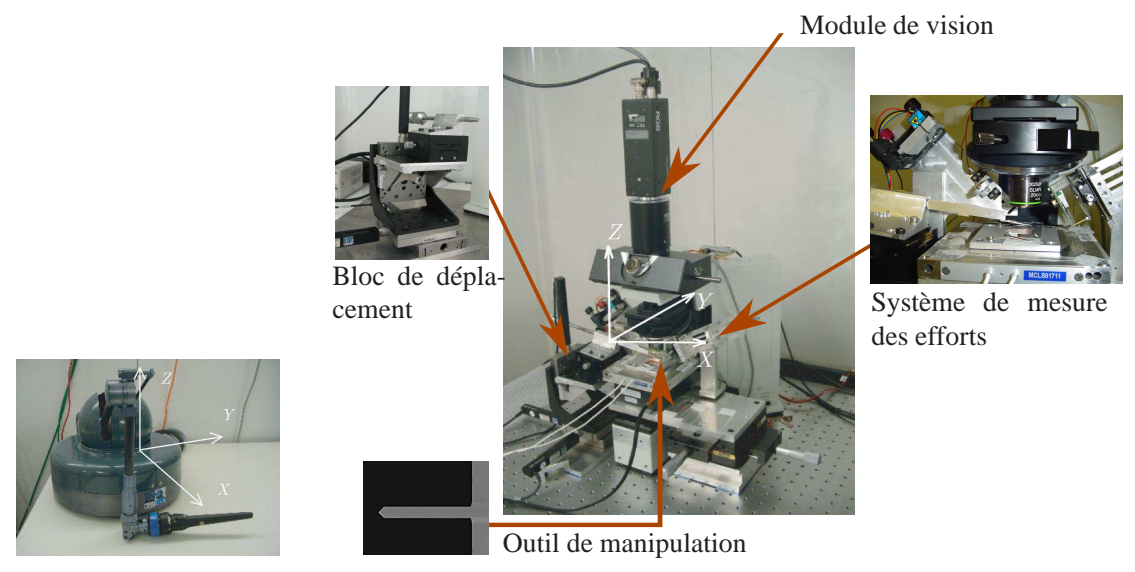

(a) interface haptique

(b) plate-forme de micro-manipulation de type Virtuose

Figure 5. Plate-forme de téléopération à l'échelle microscopique avec retour d'effort

La plate-forme utilisée pour réaliser les expériences est représentée figure 5. L'interface haptique disponible est de type Virtuose, fabriquée par Haption ${ }^{1}$ (figure 5a). C'est une interface à six degrés de liberté, avec un retour d'effort selon trois axes (les trois translations). Elle transmet des efforts de $5 \mathrm{~N}$ en continu, et de $10 \mathrm{~N}$ pour les

1. http ://www.haption.com/ 
forces maximales. L'espace de travail est de $500 \mathrm{~mm}$ selon l'axe vertical, et respectivement de 350 et $640 \mathrm{~mm}$ pour les deux autres translations. Ce robot est modélisé par un corps rigide d'inertie $M_{h}=0,4 \mathrm{~kg}$ et d'amortissement $B_{h}=0,1 \mathrm{~N} \cdot \mathrm{s} \cdot \mathrm{m}^{-1}$ (cf. équations [5] et [6]).

Le système de micro-manipulation est représenté figure 5b. Les principaux éléments sont :

- le module de vision, composé d'un microscope optique avec un objectif 20x, relié à une caméra CCD. Il permet d'estimer la position relative d'objets micrométriques dans le plan du substrat.

- le bloc de déplacement, utilisé pour manipuler le substrat. Il est composé de 3 nanotranslateurs fabriqués par Physik Instrumente ${ }^{2}$, ayant une course (respectivement résolution) de $12 \mu \mathrm{m}$ (respectivement 1,83 nm) pour l'axe vertical, et une course (respectivement résolution) de $50 \mu \mathrm{m}$ (respectivement $0,1 \mathrm{~nm}$ ) pour les axes horizontaux. La fonction de transfert des nanotranslateurs est une fonction de second ordre (cf. équations [7] et [8]), avec deux constantes de temps $\tau_{1}=1,35 \cdot 10^{-3} \mathrm{~s}$ et $\tau_{2}=0,57 \cdot 10^{-3} \mathrm{~s}$ :

- l'outil de manipulation. Il s'agit d'une poutre dont la raideur est choisie selon l'application envisagée ;

- le système de mesure des efforts par laser (cf. sous-section 2.1).

\subsection{Interaction avec le nanomonde lors d'une manipulation simple d'approche retrait}

Une poutre de raideur $k_{z}=2,4 \mathrm{~N} \cdot \mathrm{m}^{-1}$ est utilisée. Les facteurs d'échelle sont choisis tels que $A_{d}=0,05 \cdot 10^{6}$ (un déplacement de $1 \mathrm{~cm}$ de la poignée de l'interface haptique correspond à un mouvement de $0,2 \mu \mathrm{m}$ de la poutre) et $A_{f}=6 \cdot 10^{6}$ (un effort de $50 \mathrm{nN}$ sera ressenti par l'utilisateur comme une force de $0,3 \mathrm{~N}$ ). Les paramètres des correcteurs sont sélectionnés selon les résultats des analyses de transparence et de stabilité (tableau 2). Un bon compromis entre la transparence en modes contact et noncontact est de prendre $K_{f p}=100 \mathrm{~N} \cdot \mathrm{m}^{-1}$. Cette valeur est effectivement inférieure à la valeur maximale autorisée $\frac{4 M_{h}}{T_{e}^{2}}$. En choisissant $B_{f p}=2,0 \mathrm{~N} \cdot \mathrm{s} \cdot \mathrm{m}^{-1}$ la viscosité introduite dans le système est suffisante. $B_{f p}$ est bien supérieur à la valeur minimale $\frac{K_{f p} T_{e}}{2}-B_{h}=0,15 \mathrm{~N} \cdot \mathrm{s} \cdot \mathrm{m}^{-1}$, et la transparence en non contact reste bonne. $G_{n}$ vaut $G_{n}=0,90 G_{n_{l i m}}=48,0 \mathrm{~m} \cdot \mathrm{N}^{-1} \cdot \mathrm{s}^{-1}$.

Les gains choisis doivent vérifier les équations [23], [24] et [25] pour garantir que le système reste stable quel que soit l'environnement. Pour vérifier si ces conditions sont respectées, les fonctions $C_{1}, C_{2}$ et $C_{3}$ sont représentées dans le domaine fréquentiel. Les résultats sont donnés figure 6 .

2. http ://www.physikinstrumente.com/ 

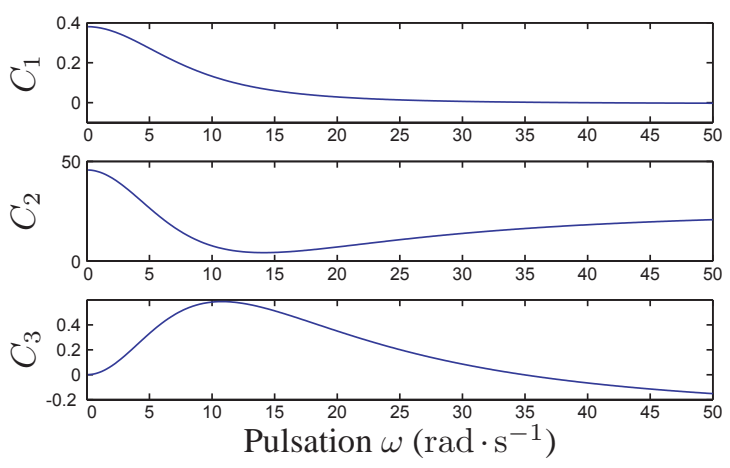

Figure 6. Critère de Llewelyn : valeurs de $C_{1}, C_{2}$ et $C_{3}$ en fonction de $\omega$

Pour les pulsations inférieures à $\omega_{c}=35 \mathrm{rad} \cdot \mathrm{s}^{-1}, C_{1}, C_{2}$, et $C_{3}$ sont positifs : le critère de Llewelyn est vérifié. Compte tenu de la bande passante du système, les pulsations supérieures à $\omega_{c}$ sont atténuées, donc le système est utilisable avec les valeurs choisies.

Pour vérifier expérimentalement que les gains choisis sont adaptés au problème posé, une opération d'interaction avec le nanomonde est effectuée. Elle consiste en une manipulation d'approche retrait (cf. sous-section 2.2) lors de laquelle l'opérateur ressent les phénomènes caractéristiques à cette échelle, dont notamment les forces attractives. Les résultats obtenus sont représentés figure 7a. Les efforts ressentis par l'utilisateur, de même que ceux mesurés par le capteur d'effort (et amplifiés d'un facteur $A_{f}$ ) sont tracés. Le système reste stable pendant toute l'expérience. De plus, même si les forces $F_{m}$ renvoyées à l'utilisateur sont calculées à l'aide du correcteur $P I$, les efforts ressentis par l'utilisateur reflètent la mesure des forces d'interaction avec l'environnement (les tracés de $F_{m}$ et $A_{f} F_{e}$ sont similaires). Les oscillations présentes sur le tracé de $F_{m}$ sont dues au couplage virtuel. Cependant, comme la bande passante de l'interface est limitée, elles ne sont pas dérangeantes pour l'utilisateur. Ainsi, même si ce schéma de commande n'est pas parfaitement transparent, le ressenti des forces est suffisamment fidèle pour que l'utilisateur puisse ressentir le phénomène de pulloff avec un pic de $1 \mathrm{~N}$ d'amplitude. L'étude réalisée précédemment permet de régler efficacement les gains.

Le phénomène de pull-in n'est pas visible sur la figure 7a. En effet, pour éviter les manipulations trop longues, $A_{d}=0,05 \cdot 10^{6}$ est choisi (i.e. une vitesse de $1 \mathrm{~cm} \cdot \mathrm{s}^{-1}$ de la poignée de l'interface haptique représente une vitesse de $0,2 \mu \mathrm{m} \cdot \mathrm{s}^{-1}$ pour le robot esclave). Compte tenu de cette valeur de $A_{d}$, la vitesse du nanotranslateur est trop élevée par rapport au phénomène de pull-in, et ce dernier ne peut donc être retourné à l'utilisateur.

Pour démontrer la robustesse de l'approche choisie par rapport à la raideur de l'environnement, la même expérience est réalisée pour des poutres de différentes raideurs. 

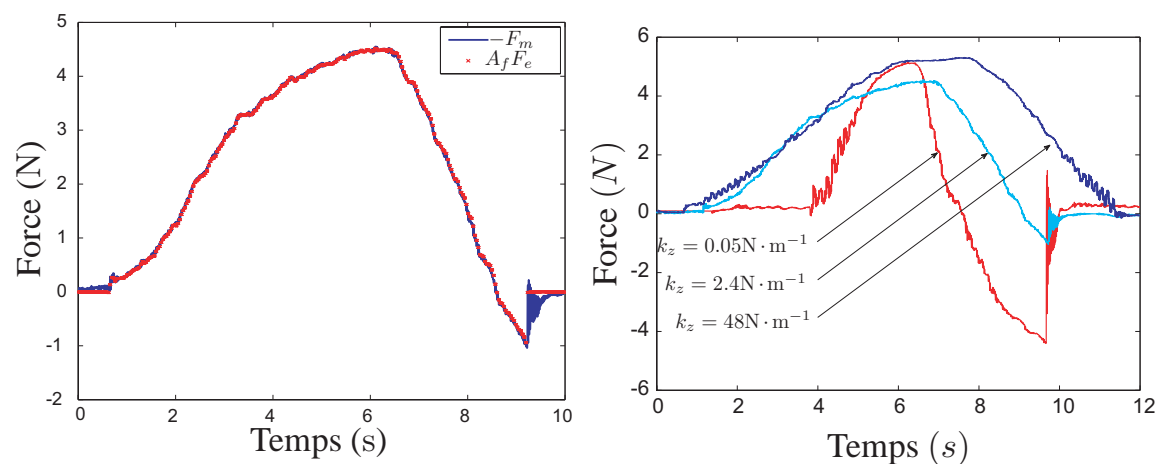

(a) poutre utilisée : $k_{z}=2,4 \mathrm{~N} \cdot \mathrm{m}^{-1}$

(b) poutres utilisées : $k_{z}=0.05 \mathrm{~N} \cdot \mathrm{m}^{-1}$ $\left(A_{d}=0.05 \cdot 10^{6}, A_{f}=6 \cdot 10^{6}\right)$

$\left(K_{f p}=100 \mathrm{~N} \cdot \mathrm{m}^{-1}, B_{f p}=1.2 \mathrm{~N} \cdot \mathrm{s} / \mathrm{m}\right.$,

$\left.G_{n}=2329 \mathrm{~m} / \mathrm{N} \cdot \mathrm{s}\right), k_{z}=2.4 \mathrm{~N} \cdot \mathrm{m}^{-1}$

$\left(K_{f p}=100 \mathrm{~N} \cdot \mathrm{m}^{-1}, B_{f p}=2.0 \mathrm{~N} \cdot \mathrm{s} \cdot \mathrm{m}^{-1}\right.$,

$\left.G_{n}=48.0 \mathrm{~m} \cdot \mathrm{N}^{-1} \cdot \mathrm{s}^{-1}\right)$ et $k_{z}=48 \mathrm{~N} \cdot \mathrm{m}^{-1}$

$\left(K_{f p}=100 \mathrm{~N} \cdot \mathrm{m}^{-1}, B_{f p}=1.5 \mathrm{~N} \cdot \mathrm{s} \cdot \mathrm{m}^{-1}\right.$,

$G_{n}=1.98 \mathrm{~m} \cdot \mathrm{N}^{-1} \cdot \mathrm{s}^{-1}$ )

Figure 7. Courbes d'approche-retrait effectuées avec le schéma de commande FP

Les résultats sont donnés figure $7 \mathrm{~b}$. Le facteur d'échelle en déplacement est identique pour l'ensemble des expériences. Celui d'amplification des efforts est choisi en fonction de la raideur de la poutre pour que l'utilisateur puisse ressentir facilement le contact. Les coefficients choisis sont donc : $A_{f}=200 \cdot 10^{6}$ pour une poutre de raideur $k_{z}=0,05 \mathrm{~N} \cdot \mathrm{m}^{-1}$, et $A_{f}=0,2 \cdot 10^{6}$ pour une raideur $k_{z}=48 \mathrm{~N} \cdot \mathrm{m}^{-1}$. Les autres gains sont déterminés en utilisant le tableau 2 . Il convient de noter que les expériences sont effectuées dans un environnement non contrôlé. Les conditions d'humidité et de température peuvent être modifiées entre les différentes expériences. Cependant, l'amplitude des pull-off obtenus est effectivement plus importante sur les courbes d'effort réalisées avec des poutres souples.

Grâce à la méthodologie de réglages des gains proposée, le schéma de commande FP est adapté aux nanomanipulations puisque le système reste stable même lors de la mise en contact avec le substrat. Cette propriété est montrée expérimentalement pour des poutres dont la raideur varie de $0,05 \mathrm{~N} \cdot \mathrm{m}^{-1}$ à $48 \mathrm{~N} \cdot \mathrm{m}^{-1}$. Des forces de $10 \mathrm{nN}$ (pull-off de la poutre de raideur $k_{z}=0,05 \mathrm{~N} \cdot \mathrm{m}^{-1}$ ) sont ressenties par l'opérateur. Un exemple de manipulation plus complexe peut donc maintenant être étudié.

\subsection{Ressenti des efforts lors d'une manipulation complexe de roulement $2 D$}

Dans cette sous-section, un exemple de téléopération 2D avec retour d'effort est proposé. Une poutre sans pointe est utilisée pour manipuler des objets. A l'aide de 
la mesure de ses déformations, et de ses propriétés mécaniques, la force appliquée sur la poutre est déterminée. Ces informations sont utilisées pour définir des indications sur l'état du système lors de la manipulation, comme notamment la position de l'objet par rapport à l'outil, qui seront transmises par retour haptique à l'opérateur. Cette approche est mise en œuvre sur une expérience de roulement de microsphères. Contrairement aux travaux de (Li et al., 2004), la force envoyée à l'utilisateur n'est pas la force réelle s'appliquant sur la poutre, mais une force modifiée, basée sur la mesure des déformations de la poutre, pour aider l'opérateur à effectuer une tâche de manipulation donnée.

\subsubsection{Mesure des déformations de la poutre}

Les déformations de la poutre sont mesurées à l'aide d'un laser comme expliqué sous-section 2.1. Ce dernier est focalisé à l'extrémité de la poutre, sur sa ligne médiane (point $E(L, 0)$ où $L$ est la longueur de la poutre) tel qu'illustré sur la figure 8 .

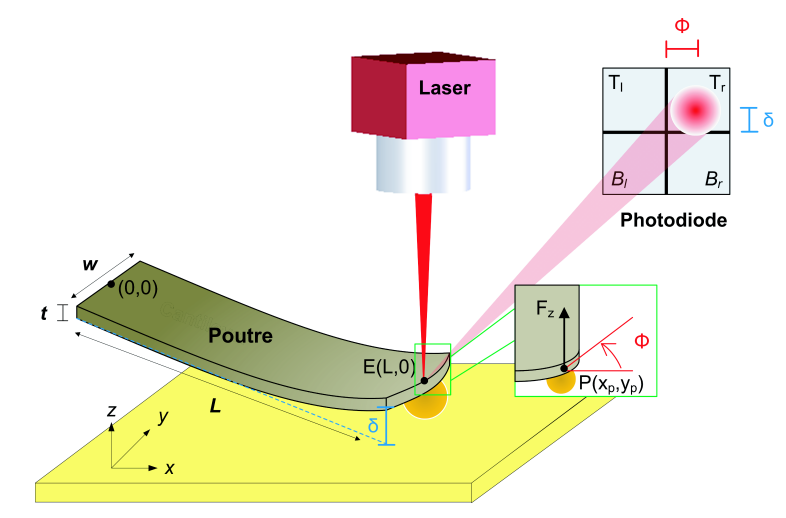

Figure 8. Principe de la mesure des déformations de la poutre à l'aide d'un laser

Une force appliquée au point $P\left(x_{p}, y_{p}\right)$ provoque une déflexion $\delta$, et une rotation $\phi$. A partir des sensibilités verticale et horizontale $S_{v}$ et $S_{h}$ calibrées de la photodiode (Langlois et al., 2007), (Cain et al., 2000) et de la mesure des tensions de sortie $u_{v}$ et $u_{h}$, il est possible de connaître les déformations de la poutre au point $E$ :

$$
\begin{aligned}
\delta(L, 0) & =S_{v} u_{v} \\
\phi(L, 0) & =S_{h} u_{h}
\end{aligned}
$$

\subsubsection{Relation entre la déformation de la poutre et les forces appliquées}

L'ensemble des paramètres relatifs à la poutre sont résumés dans le tableau 3. Les valeurs numériques sont données pour une poutre en silicium et les dimensions sont celles de l'outil utilisé sous-section 4.2.4 pour réaliser les expériences. 


\begin{tabular}{|c|c|c|}
\cline { 2 - 3 } \multicolumn{1}{c|}{} & Signification & Valeur numérique \\
\hline$L, t, w$ & $\begin{array}{c}\text { Ongueur, épaisseur et largeur de la } \\
\text { poutre }\end{array}$ & $\begin{array}{c}L=220 \mu \mathrm{m}, t=2,9 \mu \mathrm{m}, \\
w=27 \mu \mathrm{m}\end{array}$ \\
\hline$k_{z}, k_{t_{\phi}}$ & $\begin{array}{c}\text { raideurs normale et de torsion de la } \\
\text { poutre }\end{array}$ & $\begin{array}{c}k_{z}(L)=2,4 \mathrm{~N} \cdot \mathrm{m}^{-1}, \\
k_{t_{\phi}}(L)=64,0 \cdot 10^{-9} \mathrm{~N} . \mathrm{m}\end{array}$ \\
\hline$E, G$ & $\begin{array}{c}\text { module de Young et de cisaillement } \\
E=150 \mathrm{GPa}, \mathrm{G}=\frac{E}{2(1+\nu)}\end{array}$ \\
\hline$\nu$ & coefficient de Poisson & $\nu=0,17$ \\
\hline$I_{x}, I_{y}$ & $\begin{array}{c}\text { moment quadratique par rapport à } \\
\text { l'axe } x \text { (respectivement } y \text { ) }\end{array}$ & $I_{x}=\frac{t w^{3}}{12}, I_{y}=\frac{t^{3} w}{12}$ \\
\hline
\end{tabular}

Tableau 3. Variables décrivant la poutre et valeurs numériques associées (poutre en silicium). Les dimensions sont celles de la poutre utilisée sous-section 4.2.4

Lorsqu'une force $F_{z}\left(x_{p}, y_{p}\right)$ est appliquée sur la poutre au point $P\left(x_{p}, y_{p}\right)$ selon l'axe vertical (axe $z$ ), la poutre subit deux déformations différentes, une déflexion et une rotation. Dans les sections suivantes, les relations entre la force appliquée et la déformation de la poutre sont mises en évidence.

\subsubsection{Déformations selon l'axe $x$}

La force $F_{z}\left(x_{p}, y_{p}\right)$ fléchit la poutre. Pour une poutre encastrée-libre l'expression de la déflexion de la poutre $\delta\left(x, y_{p}\right)$ est :

$$
\delta\left(x, y_{p}\right)=\frac{F_{z}\left(x_{p}, y_{p}\right)}{E I_{y}}\left(x \frac{x_{p}^{2}}{2}-\frac{x_{p}^{3}}{6}\right) \text { pour } x_{p} \leq x \leq L
$$

Comme montré en sous-section 4.2.1, ce fléchissement peut être mesuré en utilisant l'équation [39]. Donc la force $F_{z}\left(x_{p}, y_{p}\right)$ peut être estimée en utilisant cette mesure :

$$
F_{z}\left(x_{p}, y_{p}\right)=k_{z}\left(x_{p}\right) \delta\left(x_{p}, y_{p}\right)
$$

où $k_{z}\left(x_{p}\right)$ est la raideur de la poutre au point $x_{p}$ :

$$
k_{z}\left(x_{p}\right)=\frac{E w t^{3}}{4 x_{p}^{3}}=\left(\frac{L}{x_{p}}\right)^{3} k_{z}(L)
$$

La raideur normale à l'extrémité de la poutre $k_{z}(L)$ est calibrée.

D'après l'équation [41] :

$$
\delta\left(x_{p}, y_{p}\right)=\frac{2 x_{p}}{3 L-x_{p}} \delta\left(L, y_{p}\right)
$$

En combinant les équations [43] et [44] on obtient :

$$
F_{z}\left(x_{p}, y_{p}\right)=\frac{2 L^{3}}{x_{p}^{2}\left(3 L-x_{p}\right)} k_{z}(L) \delta\left(L, y_{p}\right)
$$




\subsubsection{Déformation selon l'axe $y$}

Le calcul de la déformation est effectué en considérant une poutre selon l'axe $y$ (libre à son extrémité), et l'hypothèse que le problème est monodimensionnel. La force $F_{z}\left(x_{p}, y_{p}\right)$ appliquée selon la direction verticale provoque une rotation $\phi\left(x_{p}, y_{p}\right)$ de la poutre :

$$
\phi\left(x_{p}, y_{p}\right)=\frac{F_{z}\left(x_{p}, y_{p}\right)}{2 E I_{x}} y_{p}^{2}+\phi\left(x_{p}, 0\right)
$$

Cet angle est utilisé pour calculer le moment correspondant $M\left(x_{p}, y_{p}\right)$ :

$$
M\left(x_{p}, y_{p}\right)=k_{t_{\phi}}\left(x_{p}\right) \phi\left(x_{p}, y_{p}\right)
$$

où $k_{t_{\phi}}\left(x_{p}\right)=\frac{G w t^{3}}{3 x_{p}}$ est la raideur de torsion. D'après [46], on obtient :

$$
M\left(x_{p}, y_{p}\right)=k_{t_{\phi}}\left(x_{p}\right)\left[\frac{F_{z}\left(x_{p}, y_{p}\right)}{2 E I_{x}} y_{p}^{2}+\phi\left(x_{p}, 0\right)\right]
$$

Ces relations entre la force appliquée sur la poutre et la mesure de ses déformations présentée dans cette sous-section sont utilisées dans les sous-sections suivantes pour déterminer des informations utiles au sujet des forces d'interaction et pour aider l'utilisateur à effectuer la tâche de manipulation choisie.

\subsubsection{Calcul des forces renvoyées}

Une expérience de roulement est choisie pour démontrer le principe de l'utilisation d'un retour haptique pour la micromanipulation. Le roulement est en effet une manière prometteuse de manipuler les objets (billes, nanotubes de carbone) à cette échelle. Il peut également être utilisé pour déposer des objets attachés à une poutre (Haliyo et al., 2006), ou pour indenter des surfaces (Tafazzoli et al., 2006). Le retour d'effort est utilisé pour fournir à l'opérateur des indications sur la position des objets par rapport à l'outil.

Il faut noter que d'autres stratégies de manipulation, de même que des objets de tailles différentes, auraient pu être choisis. Le roulement est simplement une des applications possibles pour étudier le principe de l'utilisation du retour haptique pour fournir des renseignements sur les efforts d'interaction.

\subsubsection{Roulement longitudinal}

Dans (Haliyo et al., 2006), les auteurs affirment que pour faire rouler des objets sans glissement, un effort du même ordre de grandeur que la force de pull-off doit leur être appliqué. Comme la poutre est inclinée de quelques degrés par rapport au plan $(x, O, y)$ (cf. figure 8), la déflexion à son extrémité est maintenue constante pour appliquer cette force. Pour maintenir cette déflexion constante, la position de l'origine de la poutre est asservie. 
La position initiale de la bille sous la poutre est notée $x_{p}(0)$. Ce point, de même que la position de la poutre selon l'axe $x$ à l'instant $j, x_{c}(j)$, sont supposés être connus (estimation de la position à l'aide du module de vision). Comme le mouvement effectué est un roulement sans glissement, la position de la sphère $x_{p}$ à l'instant $j$ est estimée à partir de l'expression :

$$
x_{p}(j)=x_{p}(0)+\frac{x_{c}(j)-x_{c}(0)}{2}
$$

Pour le roulement selon l'axe longitudinal, la bille est située initialement à l'extrémité de la poutre, sur sa ligne médiane (point $E\left(x_{\max }, 0\right)$ figure 8 ). Sa position selon l'axe $y$ est maintenue constante afin que la poutre ne subisse pas de torsion : $\phi(L, 0)=0$. La poutre est ensuite déplacée selon l'axe $x$, pour faire rouler la bille. Pour fournir à l'utilisateur des informations sur la position de l'objet manipulé, la force ressentie $F_{e}^{x}$ est choisie de sorte que sa valeur soit minimale lorsque la bille est à l'extrémité de la poutre et qu'elle augmente quand la bille se rapproche de l'origine. Cette force est calculée à partir de la force normale $F_{z}$ appliquée par la bille sur la poutre. Cependant, comme $\delta\left(L, y_{p}\right)$ n'est pas disponible, $F_{e}^{x}$ est calculée par l'équation [50] :

$$
F_{e}^{x}\left(x_{p}, y_{p}\right)=\frac{2 L^{3}}{x_{p}^{2}\left(3 L-x_{p}\right)} k_{z}(L) \delta(L, 0)
$$

où $\delta(L, 0)$ est mesurée en utilisant la photodiode (cf. équation [39]).

L'allure de la force est donnée figure 9a (comme précisé ci-dessus, la position de l'origine de la poutre est asservie telle que $\delta(L, 0)$ soit constante). Cette force est effectivement minimale à l'extrémité de la poutre. De manière à ce que ce point représente une position d'équilibre pour l'utilisateur, un offset sera utilisé pour rendre la force nulle.

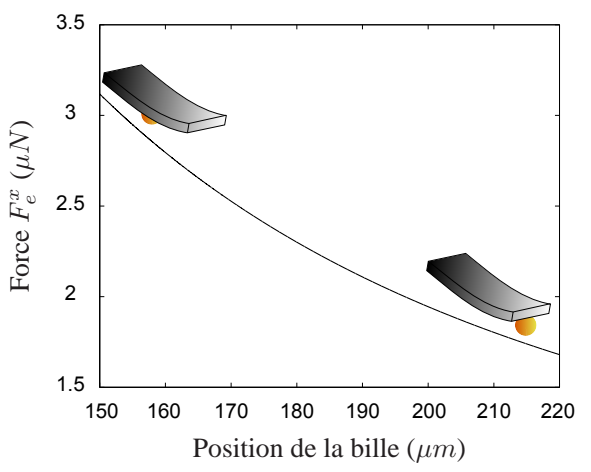

(a) axe $x$

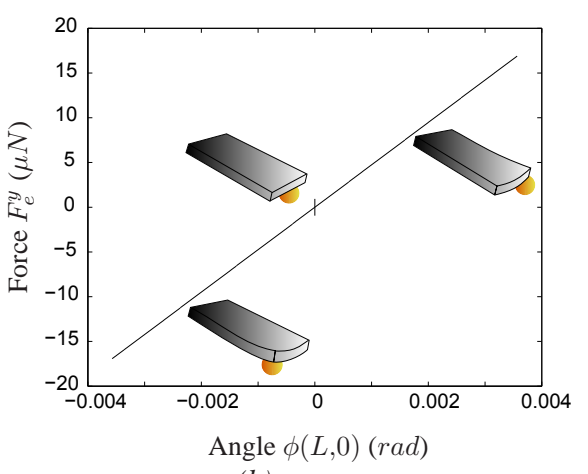

(b) axe $y$

Figure 9. Profil des efforts fournis à l'utilisateur 


\subsubsection{Roulement latéral}

Pour le roulement latéral, la position selon l'axe $x$ est constante, et la poutre est déplacée selon l'axe $y$. Lors de l'exécution de tâches de roulement, il est important de conserver la bille sur la ligne médiane de la poutre, pour ne pas perdre le contact entre l'objet et l'outil. Pour aider l'utilisateur à maintenir la bille sur cette ligne, une force $F_{e}^{y}$ lui est envoyée par l'interface haptique. Elle correspond à la force de torsion

qui tend à maintenir la poutre horizontale, $\frac{M\left(x_{p}, y_{p}\right)}{y_{p}}$. Cependant, d'après l'équation [48], la variation de cette force n'est pas facilement interprétable. Nous proposons de linéariser cette expression :

$$
F_{e}^{y}\left(x_{p}, y_{p}\right)=\frac{M(L, 0)}{\frac{w}{2}}=\frac{k_{t_{\phi}}(L)}{\frac{w}{2}} \phi(L, 0)
$$

où $\phi(L, 0)$ est la mesure de la rotation donnée par la sortie de la photodiode (cf. équation [40]). Les variations de la force $F_{e}^{y}$ sont représentées figure $9 \mathrm{~b}$.

Selon le signe de $\phi(L, 0)$, cette force est positive ou négative. Plus $\phi(L, 0)$ est important, plus l'amplitude de la force augmente. Envoyée à l'utilisateur à l'aide de l'interface haptique, cette force va en effet tendre à maintenir la sphère sur la ligne médiane de la poutre (qui est l'ensemble des points pour lesquels la rotation est nulle). Comme l'angle augmente quand le point d'application de la force $F_{z}\left(x_{p}, y_{p}\right)$ s'éloigne de la ligne médiane, et que $F_{e}^{y}$ est proportionnelle à l'angle, le retour d'effort peut également aider l'utilisateur à estimer la position de la sphère $\left(x_{p}, y_{p}\right)$ sous la poutre.

\subsubsection{Roulement $2 \mathrm{D}$}

Comme montré par les équations [50] et [51], les forces $F_{e}^{x}$ et $F_{e}^{y}$ envoyées à l'utilisateur sont découplées. Des opérations de roulement 2D peuvent être effectuées en utilisant le retour d'effort décrit dans les sous-sections 4.2.3.1 et 4.2.3.2. Les forces sont alors envoyées sur deux axes différents de l'interface haptique.

\subsubsection{Ressenti des efforts}

La stratégie décrite sous-section 4.2.3 est évaluée à l'aide d'expériences de roulement sur des billes de verre ayant un rayon de $25 \mu \mathrm{m}$.

\subsubsection{Roulement longitudinal}

Dans cette sous-section, une expérience de roulement selon l'axe $x$ de la poutre est présentée. La position verticale de la poutre est asservie de sorte que la déflexion à son extrémité soit constante $(\delta(L, 0)=0,7 \mu \mathrm{m}$, ce qui correspond à une force de $1,7 \mu N$ quand la bille est au point $E$ ).

Les valeurs des gains des correcteurs sont choisies selon le tableau 2. $A_{d}^{x}=0,05 \cdot 10^{6}$ permet d'avoir un déplacement suffisamment précis de la bille pour cette expérience. $A_{f}^{x}$ est choisi pour que la force $F_{m}^{x}$ soit de l'ordre de quelques newtons, pour être facilement détectable par un utilisateur non entraîné. Dans le cas 
du roulement sur la longueur, la force sera amplifiée d'un facteur $A_{f}^{x}=25 \cdot 10^{6}$. $K_{f p}^{x}=100 N . m^{-1}$ et $B_{f p}^{x}=0,1 N . s . m^{-1}$ sont choisis pour permettre un ressenti raide du contact, tout en supprimant les oscillations grâce à l'ajout d'amortissement. $G_{n}^{x}$ est calculé en utilisant les valeurs des gains précédents : $G_{n}=0,90 G_{n_{l i m}}$.

Au cours de l'expérience, l'utilisateur fait rouler la bille du point $x=x_{\max }=L$ (extrémité de la poutre) vers l'origine de la poutre jusqu' au point $x_{m i n}$, puis retourne à un point situé entre $x_{\min }$ et $x_{\max }$, noté $x_{i n t}$ (pour $x$ intermédiaire). L'opérateur manipule ensuite la bille d'avant en arrière autour de la position $x_{\text {int }}$. Les résultats sont donnés figure 10a. La position de la bille selon l'axe $x$, ainsi que les forces renvoyées à l'opérateur $F_{m}^{x}$ sont représentées.

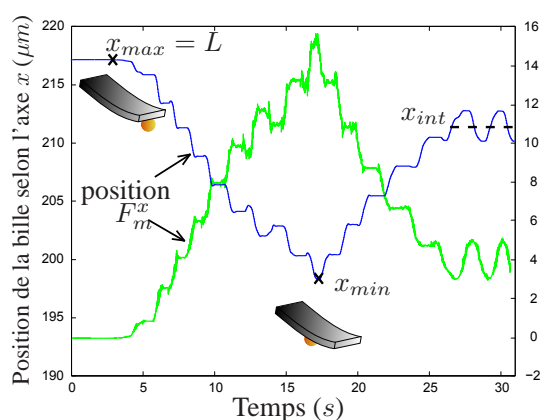

(a) roulement longitudinal. Paramètres utilisés : $A_{f}^{x}=25 \cdot 10^{6}, A_{d}^{x}=50 \cdot 10^{3}$, $K_{f p}^{x}=100 N . m^{-1}, B_{f p}^{x}=0,1$ N.s.m ${ }^{-1}$, $G_{n}^{x}=825 m \cdot N^{-1} \cdot s^{-1}$

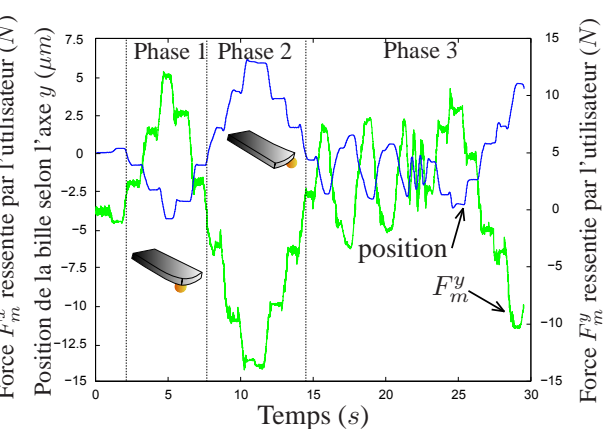

(b) roulement latéral. Paramètres utilisés: $A_{f}^{y}=2 \cdot 10^{6}, A_{d}^{y}=50 \cdot 10^{3}$,

$K_{f p}^{y}=100$ N.m ${ }^{-1}, B_{f p}^{y}=1,5$ N.s.m ${ }^{-1}$, $G_{n}^{y}=19,8 m \cdot N^{-1} \cdot s^{-1}$

Figure 10. Positions et forces pendant les expériences de roulements longitudinal et latéral

Compte tenu du facteur de déplacement $A_{d}^{x}$ utilisé, un mouvement de $1 \mathrm{~cm}$ de la poignée de l'interface haptique correspond à un mouvement de $0,2 \mu \mathrm{m}$ pour la poutre. Au cours de l'expérience, la poutre est manipulée sur environ $40 \mu \mathrm{m}$, ce qui demande un déplacement de $2 \mathrm{~m}$ de la poignée de l'interface haptique. Comme l'espace de travail du bras maître est limité (environ $35 \mathrm{~cm}$ pour l'axe $x$ ), une fonction permettant de recaler la position de l'interface haptique sans bouger la poutre est implémentée. Les paliers apparaissant sur le tracé de la position selon l'axe $x$ sont dus à ce recalage : la poutre est immobile pendant cette opération, et par conséquent, la force renvoyée à l'utilisateur est constante.

Comme montré figure 10a et prévu sous-section 4.2.3.1, la force envoyée à l'utilisateur augmente lorsque la sphère s'éloigne de l'extrémité de la poutre. Pour $x=x_{\max }$, la force est nulle. L'opérateur doit fournir un effort pour éloigner la bille de cette position d'équilibre. Le retour d'effort aide également l'utilisateur à estimer la distance entre la bille et le point $x=x_{\max }$, puisque sa valeur augmente quand la bille s'éloigne de ce point. 


\subsubsection{Roulement latéral}

Comme pour le roulement sur la longueur, la déflexion verticale de la poutre est maintenue constante. Sa position selon l'axe $x$ est également fixe.

Le facteur d'échelle en déplacement est identique à celui utilisé pour le roulement latéral (la précision désirée pour la manipulation est inchangée). Compte tenu du calcul de $F_{e}^{y}$ (moment transformé en force), son amplitude est environ 10 fois plus importante que celle de $F_{e}^{x}$. Comme il est souhaitable de renvoyer à l'utilisateur des forces $F_{m}^{x}$ et $F_{m}^{y}$ du même ordre de grandeur, le coefficient d'amplification en effort a été diminué d'un facteur 10. Le gain intégral du correcteur PI est identique à celui utilisé pour l'axe $x$. Cependant, le gain proportionnel doit être augmenté tel que $B_{f p}^{y}=1,5$ N.s.m ${ }^{-1}$, à cause de l'alternance entre les forces positives et négatives qui a lieu quand la bille passe d'un côté à l'autre de la poutre. Pour limiter les oscillations dans le système, des frottements doivent en effet être ajoutés. Comme pour le roulement latéral, $G_{n}^{y}$ est calculé selon la valeur des autres gains.

L'utilisateur déplace la poutre selon $y$. Commençant sur la ligne médiane ( $y=$ 0), il déplace la bille dans le sens négatif (phase 1), puis positif (phase 2). Phase 3, il effectue des alternances rapides entre les sens positif et négatif (cf. figure 10b). L'utilisateur ressent la force $F_{m}^{y}$, calculée à partir de $F_{e}^{y}$ (cf. équation [51]).

Le même facteur d'échelle en déplacement que dans la sous-section 4.2.4.2 est utilisé $\left(A_{d}^{y}=50 \cdot 10^{3}\right)$. Pour la même raison de limitation de l'espace de travail de l'interface haptique (la course maximale est de $64 \mathrm{~cm}$ pour l'axe $y$ ), la fonction de recalage est utilisée, et provoque des paliers sur les courbes de position et de force.

Comme prévu sous-section 4.2.3.2, l'utilisateur ressent une force qui le guide pour ramener la poutre dans une position d'équilibre (pas de torsion). Les forces renvoyées permettent à un utilisateur non entraîné de ressentir distinctement de quel côté de la poutre la sphère est située. De plus, il est vérifié expérimentalement que le système reste stable même si l'utilisateur crée de petites oscillations (cf. tracé pour $t \approx 22 \mathrm{~s}$ ).

\subsubsection{Roulement 2D}

Cette expérience consiste à combiner les roulements selon la longueur et la largeur, pour effectuer une tâche de roulement 2D avec retour d'effort. Ceci est possible puisque les signaux utilisés sont indépendants : les forces $F_{e}^{x}$ et $F_{e}^{y}$ calculées ne sont pas couplées. Elles sont donc retournées à l'utilisateur sur deux axes de l'interface.

Comme pour les roulements longitudinal et latéral, la déflexion $\delta(L, 0)$ est maintenue constante. Le mouvement effectué par l'utilisateur dans le plan $(x O y)$ est représenté figure 11a. La trajectoire est globalement circulaire, mais des formes particulières ont été ajoutées pour mieux distinguer la correspondance entre les forces retournées à l'utilisateur et le mouvement effectué. Le mouvement a été répété trois fois pour vérifier que pour une position de la bille donnée, les forces ressenties par l'utilisateur sont identiques pour les trois expériences. Les gains utilisés sont ceux donnés sous-sections 4.2.4.1 et 4.2.4.2. 


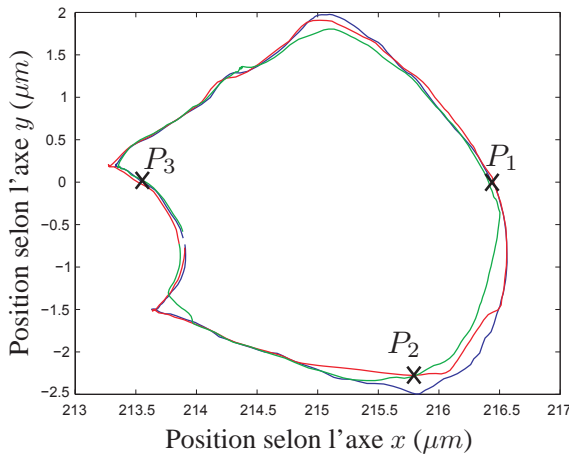

(a) positions de la sphère dans le plan $(x O y)$

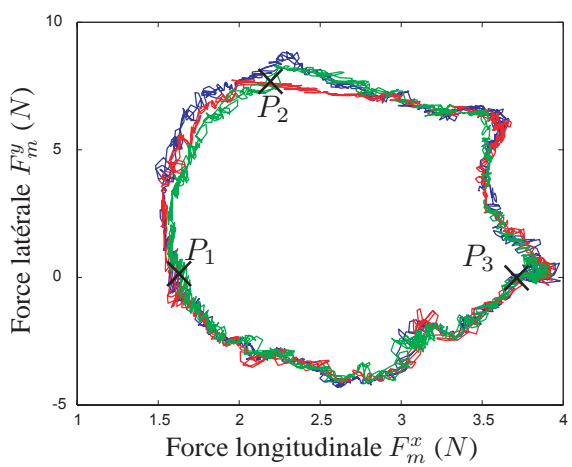

(b) forces ressenties par l'utilisateur sur les axes $x$ et $y$ de l'interface haptique

Figure 11. Positions et forces dans le plan $(x O y)$ au cours de l'expérience de roulement $2 D$

Contrairement au tracé des figures 10a et 10b, les positions selon les axes $x$ et $y$ ne présentent pas de palier. Comme les déplacements de la poutre son suffisamment petits, il n'est pas nécessaire d'utiliser la fonction de recalage.

Les forces renvoyées à l'utilisateur sont données figure $11 \mathrm{~b}$, où la force latérale est tracée en fonction de la force longitudinale. Comme l'utilisateur a effectué ces trois cercles sans atteindre l'extrémité de la poutre, la force selon l'axe $x$ n'est jamais nulle. Pour une position donnée, l'opérateur ressent la même force au cours de ces trois expériences, comme souligné par les points caractéristiques $P_{1}, P_{2}$ et $P_{3}$. Il peut donc estimer la position de la sphère sous la poutre à l'aide de ce retour d'effort.

Au cours de ces expériences, les forces ressenties par l'utilisateur lui apportent des indications sur l'état du système puisqu'il doit fournir un effort pour éloigner la bille de sa position d'équilibre (point $E$ ). Ceci est rendu possible grâce à un choix approprié des efforts retournés, et à l'utilisation d'un schéma de commande capable de renvoyer des efforts suffisamment amplifiés pour être ressentis par l'utilisateur tout en conservant un système stable. Avec le même schéma de commande, d'autres stratégies fournissant des indications à l'utilisateur à l'aide d'un retour haptique sont envisageables.

\section{Conclusion}

Le schéma de couplage force-position est adaptable à des applications nanorobotiques. Cette étude a mis en évidence une forte corrélation entre les gains des correcteurs et les performances du schéma de commande. La valeur de ces gains dépend notamment de l'environnement avec lequel l'utilisateur interagit. Bien que ce couplage haptique privilégie la stabilité et l'intuitivité au détriment de la transparence, il permet d'interagir avec le nanomonde (ressenti de phénomènes de quelques nanonew- 
tons d'amplitude), et de réaliser des opérations de roulement avec un retour d'effort bidimensionnel. D'autres opérations de manipulation avec retour d'effort sont envisageables, en utilisant des objets différents ou de nouvelles stratégies de manipulation.

Dans ce travail, de nombreuses approximations ont été faites notamment sur les paramètres de l'interface haptique, du nanotranslateur, et de la poutre lors de l'identification et du calibrage, ou encore lors de la modélisation de l'interaction poutresubstrat. Ces approximations ont permis de mieux comprendre l'influence de chacun des paramètres à l'aide d'une modélisation simple. Dans un second temps il sera intéressant de prendre en compte ces approximations en les introduisant sous la forme d'incertitudes dans le modèle pour démontrer la robustesse du schéma de commande.

La connaissance de l'influence des gains des correcteurs du schéma de commande permettra par la suite de modifier les valeurs de ces gains au cours d'une expérience. Cette stratégie sera réalisée en fonction des caractéristiques de l'environnement. L'objectif est de fournir à l'utilisateur le retour d'effort correspondant le mieux à son application, mais également aux différentes situations rencontrées au cours d'une même opération. Dans ce travail, seuls des correcteurs simples ont été utilisés (correcteurs $P$ et $P I$ ). Cependant, ce ne sont pas les plus adaptés pour modifier les gains au cours des expériences. La formulation des différentes spécifications en termes de performances du type stabilité $H_{\infty}$ ou $H_{2}$ est à envisager.

Enfin il conviendra de définir le profil de force le plus adapté lors d'opérations complexes de manipulation à l'aide de tests utilisateurs.

\section{Notations}

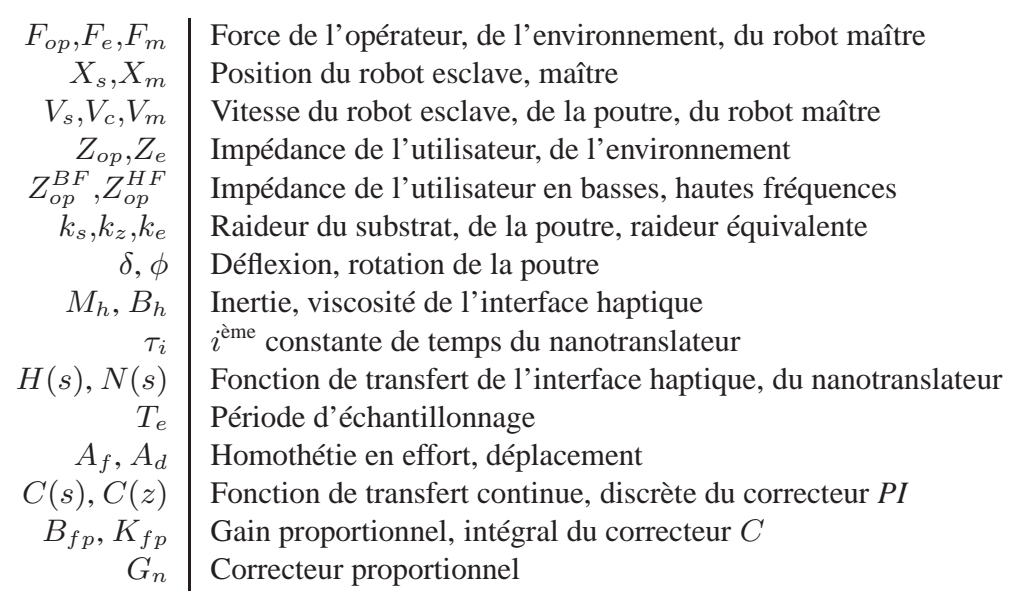

\section{Remerciements}

Ce travail a été financé par l'ANR, à travers le projet PACMAN. 


\section{Bibliographie}

Adams R., Hannaford B., « Stable haptic interaction with virtual environments », IEEE Transactions on Robotics and Automation, vol. 15, $\mathrm{n}^{\circ}$ 3, p. 465-474, 1999.

Cain R. G., Biggs S., Page N. W., « Force calibration in lateral force microscopy », Journal of Colloid and Interface Science, vol. 227, p. 55-65, 2000.

Ferreira A., Mavroidis C., « Virtual reality and haptics for nanorobotics », IEEE Robotics and Automation Magazine, vol. 13, n 3, p. 78-92, 2006.

Gil J. J., Avello A., Rubio A., Flórez J., « Stability analysis of a 1 DOF haptic interface using the Routh-Hurwitz criterion », IEEE Transactions on Control Systems Technology, vol. 12, $\mathrm{n}^{\circ}$ 4, p. 583-588, 2004.

Haliyo D. S., Dionnet F., Régnier S., « Controlled rolling of micro objects for autonomous micro manipulation », International Journal of Micromechatronics, vol. 3, n 2, p. 75-101, 2006.

Hokayem P. F., Spong M. W., « Bilateral teleoperation : an historical survey », Automatica, vol. $42, \mathrm{n}^{\circ} 12$, p. $2035-2057,2006$.

Khalil H. K., Nonlinear systems, vol. 3ème édition, Prentice Hall, 2002.

Langlois E. D., Shaw G. A., Kramar J. A., Pratt J. R., Hurley D. C., « Spring constant calibration of atomic force microscopy cantilevers with a piezosensor transfer standard », Review of Scientific Instruments, vol. 78, n 9, p. 093705, 2007.

Lawrence D., « Stability and transparency in bilateral teleoperation », IEEE Transactions on Robotics and Automation, vol. 9, n 5, p. 624-637, 1993.

Li G., Xi N., Yu M., Fung W.-K., « Development of augmented reality system for AFM-based nanomanipulation », IEEE/ASME Transactions on Mechatronics, vol. 9, n 2, p. 358-365, June, 2004.

Llewellyn F., « Some fundamental properties of transmission systems », Proceedings of the IRE, vol. 40, n 3, p. 271-283, 1952.

Onal C., Ozcan O., Sitti M., « Automated 2-D nanoparticle manipulation with an atomic force microscope », IEEE International Conference on Robotics and Automation, p. 1814-1819, 2009.

Sitti M., « Survey of nanomanipulation systems », IEEE Conference on Nanotechnology, p. 7580, 2001.

Sitti M., « Atomic force microscope probe based controlled pushing for nanotribological characterization », IEEE/ASME Transactions on Mechatronics, vol. 9, n 2, p. 343-349, 2004.

Sitti M., « Microscale and nanoscale robotics systems [Grand Challenges of Robotics] », IEEE Robotics and Automation Magazine, vol. 14, n 1, p. 53-60, 2007.

Tafazzoli A., Pawashe C., Sitti M., « Force-controlled microcontact printing using microassembled particle templates », p. 263-268, 2006.

Xie H., Vitard J., Haliyo S., Régnier S., « Calibration and nonlinearity compensation for force application in AFM based nanomanipulation », IEEE/RSJ International Conference on Intelligent Robots and Systems, p. 4024-4029, 2008. 